\title{
KETJU: Post-Newtonian-Accurate Supermassive Black Hole Dynamics in GADGET-3
}

\author{
Antti Rantala, Pauli Pihajoki and Peter H. Johansson \\ Department of Physics, University of Helsinki \\ Gustaf Hällstrmin katu 2A,00560, Helsinki,Finland \\ email: antti.rantala@helsinki.fi
}

\begin{abstract}
We present KETJU, a new regularized tree code based on algorithmic chain regularization and implemented into Gadget-3. This new code is able to follow simultaneously galactic-scale dynamical and astrophysical processes and the small-scale supermassive black hole binary dynamics. We present here the general idea of this new code and show a test simulation of black hole binary dynamics in a galaxy merger of two massive elliptical galaxies. The separation of the black holes at the time of the merger is several orders of magnitude smaller in KETJU than when compared to ordinary Gadget-3 simulations. The merger timescale is also longer by $100-200$ Myr.
\end{abstract}

Keywords. black hole physics, galaxies: kinematics and dynamics, galaxies: nuclei, methods: n-body simulations

\section{Introduction}

All massive early-type galaxies and stellar bulges host supermassive black holes (SMBH) in the mass range of $10^{6} M_{\odot} \lesssim M_{\bullet} \lesssim 10^{10} M_{\odot}$ in their centres (e.g. Kormendy \& Richstone 1995). In addition, galaxies do not live in isolation but frequently interact and merge with each other shaping their evolution (White \& Rees 1978, Johansson et al. 2009). Galaxy mergers also bring multiple SMBHs into the same stellar system. As very close quasar pairs appear to be observationally rare there should be an efficient physical mechanism which drives the two SMBHs rapidly into coalescence. Indeed, dynamical friction from the stars and gas in the host galaxy causes the SMBHs to sink to the center of the merger remnant. Forming a wide $(a \sim 10 \mathrm{pc})$ binary SMBH, the black holes now interact with the central stars slingshotting them away in three-body encounters. In this process the binary loses orbital energy at the expense of the ejected stars and becomes 'harder'. At sub-parsec separations, the energy loss by gravitational wave (GW) emission drives the binary into coalescence (e.g. Merritt \& Milosavljević 2005).

Future space-borne GW detection experiments, such as LISA (Amaro-Seoane et al. 2012), have generated interest in estimating the SMBH merger rates using numerical simulations. Naively, the SMBH merger rate depends on the frequency of galaxy mergers containing multiple SMBHs and the SMBH merging timescale after the binary has formed. However, there are considerable uncertainties in the estimates, especially in the duration of the slingshot-hardening phase. In the hardening phase, the inverse semi-major axis of the binary is expected to evolve as $d(1 / a) / d t \propto \rho_{\star}(t) / \sigma_{\star}(t)$, where $\rho_{\star}$ and $\sigma_{\star}$ are the stellar density and velocity dispersion at the center of the galaxy (Quinlan 1996). Thus, a simulation predicting the SMBH merger timescale should accurately describe both the global kiloparsec-scale evolution of the host galaxy of the SMBH binary and 
also model the SMBH binary evolution at sub-parsec scales. In this proceedings contribution, we present a new regularized tree code KETJU, based on algorithmic chain regularization and implemented into the widely used galaxy simulation code Gadget-3 (Springel 2005).

\section{Regularization}

\subsection{Background}

In the case of point-like simulation particles, the Newtonian gravitational force is manifestly divergent when two particles collide: the gravitational force $\left|\bar{F}_{i j}\right| \rightarrow \infty$ as their separation $\left|\bar{r}_{i}-\bar{r}_{j}\right| \rightarrow 0$. The most straightforward manner to circumvent this collision problem is to introduce a small softening length $\epsilon$ in order to render $\left|\bar{F}_{i j}\right|$ finite at particle collisions and close encounters. However, this procedure has the unfortunate consequence that the softening scale $\epsilon$, typically $1 \mathrm{pc}<\epsilon<100 \mathrm{pc}$ in modern galactic-scale simulations, becomes the natural spatial resolution limit of the simulation. This is highly undesirable as we want to simulate SMBH binary evolution at sub-parsec scales until the GW-driven coalescence. Another possibility is the regularization of the close encounters between simulation particles to avoid the Newtonian force divergences. The Keplerian two-body problem was regularized a century ago by the pioneering work of Levi-Civita. A practical three-dimensional KS-regularization method including external perturbing forces was developed by Kustaanheimo \& Stiefel (1965). Subsequent development in the field yielded several new regularization methods, such as the Aartseth-Zare method and Heggie's global regularization scheme (see e.g. Aarseth 1999 and references therein). An important chain-based formulation of the KS regularization was found by Mikkola \& Aarseth (1993). Finally, the work of Mikkola \& Tanikawa (1999) and Preto \& Tremaine (1999) on time-transformed regularization methods resulted in the formulation of the Algorithmic Regularization Chain (ARCHAIN) algorithm, which is also our choice of regularization method for KETJU ('ketju'='chain' in Finnish).

\subsection{The ARCHAIN algorithm}

The inner workings of the ARCHAIN algorithm consist of three aspects. First, the equations of motion are time-transformed. As opposed to regularization methods reviewed in the previous section, no spatial coordinate transformation is performed: the singularities remain in the equations of motion but are avoided algorithmically by using a leapfrog integrator, hence the name of the algorithm. The new time coordinate, which is an independent variable, is defined as

$$
d s=[\alpha U+\beta \Omega+\gamma] d t,
$$

where $U$ is the negative of the gravitational potential energy and the function $\Omega$ is generally used to regularize the encounters between particles with very different masses, if an extrapolation method is not used. The triplet $(\alpha, \beta, \gamma)$ determines the exact regularization procedure. Note that constants $\alpha$ and $\beta$ are dimensional so that the expression inside the brackets is dimensionless. See Mikkola \& Merritt (2006) and Mikkola \& Merritt (2008) for a detailed description of the time transformation and the different parameter choices. The equations of motion are now transformed using the new time coordinate $d s$. The algorithm also allows for Post-Newtonian corrections as velocity-dependent perturbations. The corrections are added up to the order PN3.5. The second aspect of ARCHAIN is the use of chained inter-particle vectors for each chain subsystem instead of a global reference frame. This proceduce greatly reduces the numerical round-off error 


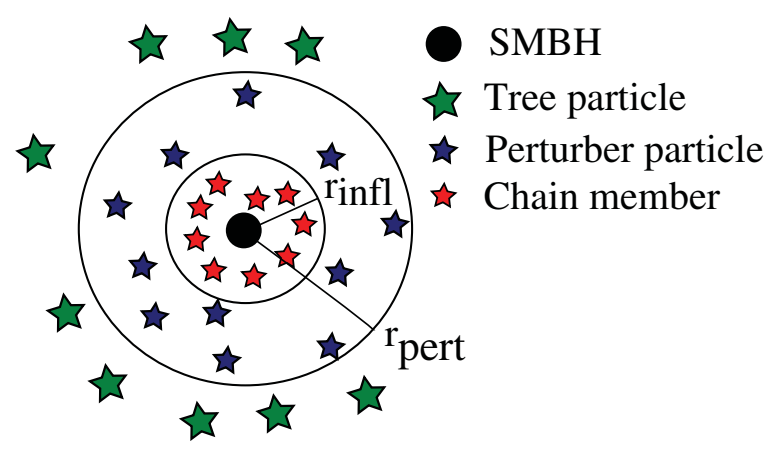

Figure 1. A schematic illustration of a chain subsystem surrounding a SMBH in the KETJU code.

even without the time transformation. Finally, an extrapolation algorithm is applied in order to push the error in the dynamical variables to a user-defined level. We utilize the Gragg-Bulirsch-Stoer (GBS) extrapolation method. The central idea of the GBS extrapolation here is to compute time evolution of the dynamical variables during a time interval $H$ with a different number of subdivision counts $n$ and finally extrapolate the variable values to $n \rightarrow \infty$. This procedure, although somewhat time-consuming, ensures that the numerical errors are kept at a very low level which is essential in computing the temporal evolution of chain systems containing SMBHs and surrounding stars.

\section{Implementation}

\subsection{Chain subsystems and perturber particles}

In developing KETJU (Rantala et al. 2016, in prep.) we follow and extend the ideas behind the rVINE code of Karl et al. (2015). In Gadget-3, the Newtonian gravitational force between the simulation particles is computed using a TreePM algorithm, including gravitational softening. We regularize the gravitational dynamics around the SMBHs in the following way. First, we give every SMBH a parametrized influence radius which is proportional to the mass of the SMBH: $r_{\text {infl }} \propto \alpha M_{\bullet}$. Every stellar particle inside the influence radius of a SMBH belongs to the chain subsystem of the particular SMBH. If the volumes of several subsystems overlap, the subsystems are merged. The dynamics of the particles in the chain (chain particles hereafter) is computed using the algorithmic chain integrator as opposed to the ordinary Gadget-3 particles (tree particles hereafter). During the chain integration, the chain particles feel the tidal perturbation of the tree particles which lie within the perturber radius $r_{\text {pert }}=\gamma r_{\text {infl }}\left(m / M_{\bullet}\right)^{1 / 3}$, where $m$ is the mass of the perturber particle and $\gamma$ is an adjustable free parameter. A simplified illustration of the situation is presented in Figure 1. The chain subsystem itself is treated as a single 'macro' particle in the tree code. Both the macro particle and the neighbouring perturber particles receive a correction to their gravitational accelerations due to the internal structure of the chain subsystem. The macro particles are set to be always at the lowest active timestep level in the tree code and the tree/chain memberships of the particles are updated every tree timestep.

\subsection{A new SMBH merging criterion}

Traditionally, two SMBHs are merged together in softened tree codes, such as Gadget-3, if their smoothing lengths overlap and their relative velocity is less than a fraction times the local sound speed. For typical resolutions employed in galaxy-scale simulations this 

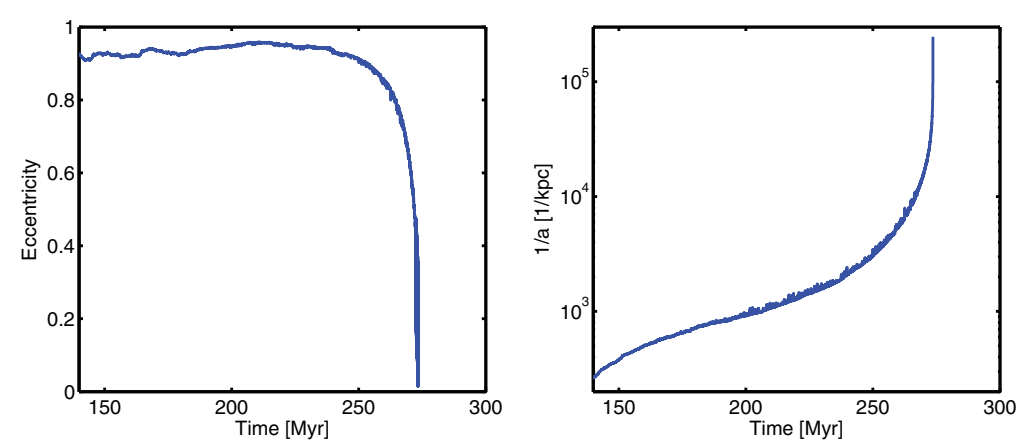

Figure 2. The evolution of eccentricity (left panel) an the inverse semi-major axis (right panel) of the SMBH binary until the GW-driven coalescence. The binary circularizes and hardens as the gravitational waves carry energy and angular momentum away from the system. The separation at the time of the SMBH merger is $\sim 1000 \mathrm{AU}$ in this particular test run.

amounts usually to $\sim 10$ pc. With KETJU, we can follow the SMBH binary to much smaller separations and thus a new SMBH merging criterion is required. The new criterion is based on the analytical GW coalescence timescale by Peters \& Mathews (1963) formula valid at PN2.5. The estimate of the merging timescale is $t_{c}=a / 4 \dot{a}$, where $\dot{a}$ is obtained from the Peter's formula:

$$
\dot{a}=-\frac{64}{5} \frac{G^{3} M_{\bullet, 1} M_{\bullet, 2}\left(M_{\bullet, 1}+M_{\bullet, 2}\right)}{c^{5} a^{3}} \frac{1+\frac{73}{24} e^{2}+\frac{37}{96} e^{4}}{\left(1-e^{2}\right)^{7 / 2}},
$$

where $M_{\bullet, 1}$ and $M_{\bullet, 2}$ are the masses of the two SMBHs and $e$ is the constant eccentricity. The timescale estimate is accurate enough for our purposes as $e_{\text {final }} \sim 0.01<<1$. See e.g. Maggiore (2007) for a more rigorous timescale estimate. The SMBHs coalesce if $t_{c}$ is smaller than the next global Gadget-3 timestep. The Post-Newtonian evolution of a SMBH binary at the late stages of a gasless ('dry') merger of two massive elliptical galaxies $\left(M_{\star}=10^{11} M_{\odot}, M_{\mathrm{DM}}=10^{13} M_{\odot}\right.$, Hernquist scale radius $\left.a=1.5 \mathrm{kpc}\right)$ is shown in Figure 2. Due to the high eccentricity of the binary inherited from the merger geometry of the merger progenitor galaxies the GW emisson drives the SMBHs to coalescence $\sim 150$ Myr after the formation of the binary. The separation at the time of the SMBH merger was $r \sim 0.005 \mathrm{pc} \sim 1000 \mathrm{AU}$ in this particular simulation.

\section{Conclusions}

We have developed a new regularized tree code KETJU using algorithmic chain regularization and implemented into Gadget-3. Using KETJU, we are able to follow the evolution of a SMBH binary in a galactic-scale simulation until the GW-driven coalescence and study simultaneously the structural evolution of the host galaxy affected by the evolution of the SMBH binary.

\section{References}

Aarseth, S. J. 1999, PASP, 111, 1333

Amaro-Seoane, P., Aoudia, S., Babak, S., et al. 2012, Classical and Quantum Gravity, 29, 124016 Johansson, P. H., Naab, T., \& Burkert, A. 2009, APJ, 690, 802

Karl, S. J., Aarseth, S. J., Naab, T., Haehnelt, M. G., \& Spurzem, R. 2015, MNRAS, 452, 2337 Kormendy, J. \& Richstone, D. 1995, ARAA, 33, 581

Kustaanheimo, P. \& Stiefel, E. 1965, J. Reine Angew. Math, 218, 204

Maggiore, M. 2007, Gravitational Waves: Volume 1: Theory and Experiments

Merritt, D. \& Milosavljević, M. 2005, Living Reviews in Relativity, 8, 
Mikkola, S. \& Valtonen, M. J. 1992, MNRAS, 259, 115

Mikkola, S. \& Aarseth, S. J. 1993, Celestial Mechanics and Dynamical Astronomy, 57, 439

Mikkola, S. \& Tanikawa, K. 1999, MNRAS, 310, 745

Mikkola, S. \& Merritt, D. 2006, MNRAS, 372, 219

Mikkola, S. \& Merritt, D. 2008, AJ, 135, 2398

Preto, M. \& Tremaine, S. 1999, AJ, 118, 2532

Peters, P. C. \& Mathews, J. 1963, Physical Review, 131, 435

Quinlan, G. D. 1996, New Astronomy, 1, 35

Springel, V. 2005, MNRAS, 364, 1105

White, S. D. M. \& Rees, M. J. 1978, MNRAS, 183, 341 